\title{
Quality-Based Registration and Reconstruction of Optical Tomography Volumes
}

\author{
Wolfgang Wein ${ }^{2,1}$, Moritz Blume ${ }^{1}$, Ulrich Leischner ${ }^{3}$, \\ Hans-Ulrich Dodt ${ }^{3}$, and Nassir Navab ${ }^{1}$ \\ ${ }^{1}$ Chair for Computer Aided Medical Procedures (CAMP) \\ Technische Universität München, Germany \\ $\{$ wein, blume, navab\}@cs.tum.edu \\ ${ }^{2}$ Imaging \& Visualization Department \\ Siemens Corporate Research, Princeton, NJ, USA \\ wolfgang. wein@siemens. com \\ ${ }^{3}$ Max Planck Institute of Psychiatry, Munich, Germany \\ \{leischner, dodt\}@mpipsykl.mpg.de
}

\begin{abstract}
Ultramicroscopy, a novel optical tomographic imaging modality related to fluorescence microscopy, allows to acquire cross-sectional slices of small specially prepared biological samples with astounding quality and resolution. However, scattering of the fluorescence light causes the quality to decrease proportional to the depth of the currently imaged plane. Scattering and beam thickness of the excitation laser light cause additional image degradation. We perform a physical simulation of the light scattering in order to define a quantitative function of image quality with respect to depth. This allows us to establish 3D-volumes of quality information in addition to the image data. Volumes are acquired at different orientations of the sample, hence providing complementary regions of high quality. We propose an algorithm for rigid 3D-3D registration of these volumes incorporating voxel quality information, based on maximizing an adapted linear correlation term. The quality ratio of the images is then used, along with the registration result, to create improved volumes of the imaged object. The methods are applied on acquisitions of a mouse brain and mouse embryo to create outstanding three-dimensional reconstructions.
\end{abstract}

\section{Introduction}

Ultramicroscopy 1 denotes a microscopical technique where the sample is illuminated from the side, perpendicular to the direction of observation (figure 11). It combines the concept of fluorescence microscopy with a procedure that makes biological tissue transparent 233. The principle of the latter is to replace the water contained in the sample by a liquid of the same refractive index as the proteins and lipids. Therefore scattering effects can be minimized and the transparency of the sample is regained; optical imaging deep inside the biological tissue is then possible. The microscope's focal plane, arbitrarily placed within the sample, is sideways illuminated with an Argon laser. Only the fluorescent light

N. Ayache, S. Ourselin, A. Maeder (Eds.): MICCAI 2007, Part II, LNCS 4792, pp. 718 725, 2007.

(C) Springer-Verlag Berlin Heidelberg 2007 


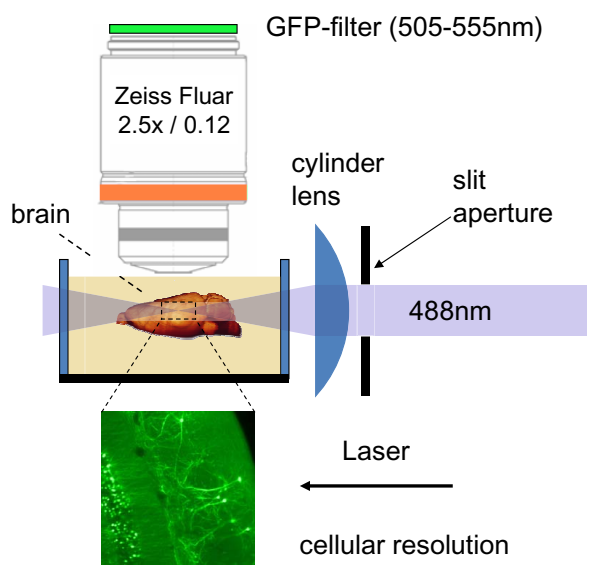

Fig. 1. Imaging setup of the Ultramicroscopy system

(generated by autofluorescence of the tissue) is measured through the microscope with a GFP-filter (505-555 nm wavelength range), and stored by a digital camera (resolution 1392 x 1024, 12 Bit grayscale). A micropositioning device advances the tray with the sample in steps of $12 \mu \mathrm{m}$, hence a stack of slices is recorded. The resulting data is a comprehensive three-dimensional reconstruction with approximate isotropic voxel size of $10 \mu \mathrm{m}$. This imaging modality allows the 3D-recording of large biological samples $(>1 \mathrm{~mm})$ with micrometer resolution, where practically no technique existed yet. A great number of biological research projects can benefit from it.

Due to tissue inhomogenity, the fluorescent light is still scattered to some extent while passing through the substance. Hence lower slices suffer a blurring effect, in relation to the distance that light travels through the object to the microscope. Our approach to overcome this problem is to acquire volumes with different orientations of the sample, while establishing corresponding volumes with quality information at the same time. This quality information will be used for both spatial registration of the different recordings, as well as the reconstruction of improved volumes disposed of blurring.

\section{Quality Function}

We want to establish a function

$$
Q: \Omega \rightarrow[0 . .1] ; \quad \Omega \subset \mathbb{R}^{3}
$$

which returns the relative quality at any position in the image space $\Omega$. It is determined by the amount of scattering of the measured light, which in turn depends on the depth that the light is traveling through the object. Assuming that light is only being scattered in the sample and not the surrounding liquid, we can reduce our problem to computing the amount of scattering with respect 

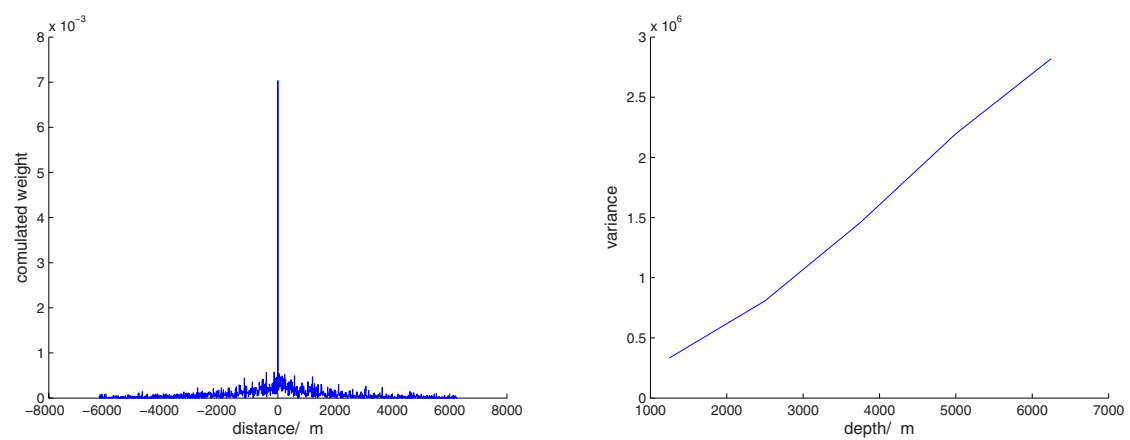

Fig. 2. Results of simulated scattering and function of standard deviation per depth

to tissue depth. This is done using a Monte Carlo simulation of light propagation similar to 4, which we briefly describe in the following.

Instead of tracing single photons, we consider photon packets with a certain initial weight for efficiency. We assume that "centers" where both scattering and absorption occur, are distributed uniformly throughout the tissue. Photon packets are initialized with the emitting position $\boldsymbol{x}_{\mathbf{0}}=(0,0,-z)^{T}$ and weight $w=1$. Then they repeatedly travel from their actual position $\boldsymbol{x}_{\boldsymbol{i}}$ a certain distance $s_{i}$ in direction $\boldsymbol{d}_{\boldsymbol{i}}$, until a scattering and absorption event occurs. The photon absorption obeys the classical attenuation relationship

$$
N(s)=N_{0} e^{-\mu_{t} s}
$$

where $\mu_{t}$ is the transmission coefficient, $\mathrm{N}(\mathrm{s})$ is the number of photons remaining at distance $s$ from an original number $N_{0}$. An adequate generating function $g(x)$ for the probability variable $s$ from a uniformly distributed variable $X$ is

$$
g(x)=\frac{1}{\mu_{t}} \log (1-x)
$$

The mean free pathlength is $\langle s\rangle=1 / \mu_{t}$. The scattering in tissue can be characterized by the Henyey-Greenstein phase function, which is a probability density function of the scattering angle, given an anisotropy factor $g$ :

$$
f_{H G}(\phi)=\frac{1-g^{2}}{4 \pi\left(1+g^{2}-2 g \cos \phi\right)^{\frac{3}{2}}}
$$

For our simulation it needs to be transformed to a generating function from a uniformly distributed random variable $X$ as well:

$$
\cos \phi=\frac{1}{2 g}\left(1+g^{2}-\left(\frac{1-g^{2}}{1-g+2 g X}\right)^{2}\right)
$$


In each iteration, the photon position, orientation and weight is then updated:

$$
\begin{array}{r}
\boldsymbol{x}_{\boldsymbol{i}+\mathbf{1}}=\boldsymbol{x}_{\boldsymbol{i}}+s_{i} \boldsymbol{d}_{\boldsymbol{i}} ; \quad w_{i+1}=w-\frac{\mu_{a}}{\mu_{t}} ; \quad \boldsymbol{d}_{\boldsymbol{i}}=\left(d_{x}, d_{y}, d_{z}\right)^{T} \\
\boldsymbol{d}_{\boldsymbol{i}+\mathbf{1}}=\left(\begin{array}{c}
\frac{\sin \theta}{\sqrt{1-d_{z}^{2}}}\left(d_{x} d_{z} \cos \phi-d_{y} \sin \phi\right)+d_{x} \cos \theta \\
\frac{\sin \theta}{\sqrt{1-d_{z}^{2}}}\left(d_{y} d_{z} \cos \phi+d_{x} \sin \phi\right)+d_{y} \cos \theta \\
-\sin \theta \cos \phi \sqrt{1-d_{z}^{2}}+d_{z} \cos \theta
\end{array}\right)
\end{array}
$$

The simulation is terminated if the photon packet reaches the top of the object, $x_{3} \geq 0$, where $\boldsymbol{x}_{\boldsymbol{i}}=\left(x_{1}, x_{2}, x_{3}\right)^{T}$. If the weight falls below a threshold $w_{i}<w_{T}$, a roulette approach decides if the photon packet is terminated. With a defined probability $p_{t}$ the photon packet is discarded, otherwise it is reinserted in the simulation with a new weight $w_{0}=w_{i} / p_{t}$. This makes sure that the energy conservation is not violated.

We are interested in the distance of the virtual point, where the light seems to come from assuming a straight line through the image plane, to the point where the simulation was started. The variance of this distance for many photon packets directly relates to the amount of blurring, i.e. our sought-after quality. Figure 2 depicts the deviation results for a simulation at particular depth, as well as the function of quality versus depth. The latter is approximately a linear relationship, which we accordingly use for assembling volumes of quality information $Q(x)$.

\section{Quality-Based Registration and Merging}

For multiple acquisitions, the preparation is carefully re-oriented within the test tube. No significant deformations occur in this context, however the coordinate system of the second acquisition has to be mapped onto the first one with very high precision, in order to use the combined information for reconstruction. This alignment is hence performed using an automatic rigid intensity-based registration method [5]. Such methods conduct a non-linear optimization of the transformation parameters, in order to maximize a similarity criterion defined on the voxel intensities of the reference and template volumes $R$ and $T$, respectively:

$$
\phi_{\text {reg }}=\arg \max _{\phi} S\left(\left\{\left(R\left(\boldsymbol{x}_{\boldsymbol{i}}\right), T\left(\phi\left(\boldsymbol{x}_{\boldsymbol{i}}\right)\right)\right) \mid \boldsymbol{x}_{\boldsymbol{i}} \in \Omega_{\phi}\right\}\right)
$$

where $\left\{\boldsymbol{x}_{\boldsymbol{i}}\right\}$ are all discrete voxel positions of the reference volume, $\phi$ is a 6 -DOF rigid transformation, and $\Omega_{\phi}$ is the volume overlap region for a given $\phi$. We use Normalized Cross-Correlation (NCC) as similarity criterion, which has been used extensively for registration of $3 \mathrm{D}$ volumes arising in medical imaging $[5]$.

$$
\begin{array}{r}
r_{i}^{\prime}=r_{i}-\bar{r} ; \quad t_{i}^{\prime}=t_{i}-\bar{t} \\
S=\frac{\sum_{i} r_{i}^{\prime} t_{i}^{\prime}}{\sqrt{\sum_{i} r_{i}^{\prime 2} \sum_{i} t_{i}^{\prime 2}}}
\end{array}
$$

For all voxels $r_{i}=R\left(\boldsymbol{x}_{\boldsymbol{i}}\right)$ of the reference volume, the corresponding voxel $t_{i}=$ $T\left(\phi\left(\boldsymbol{x}_{\boldsymbol{i}}\right)\right)$ is trilinearly interpolated from the template volume. $\bar{r}$ and $\bar{t}$ are the mean values of all reference and template intensities, respectively. 


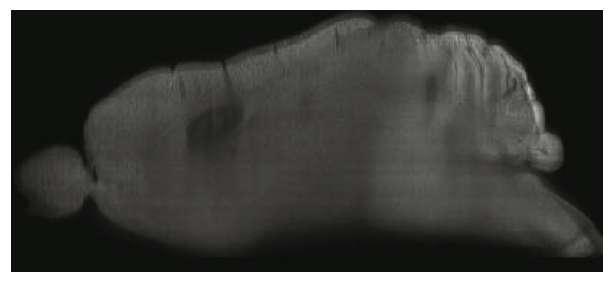

(a) Reference Volume

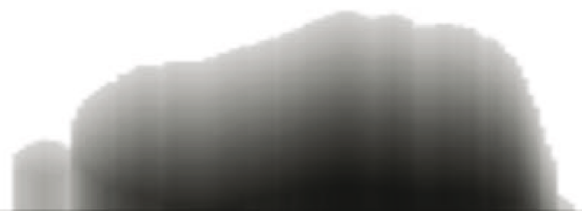

(c) Reference Quality Volume

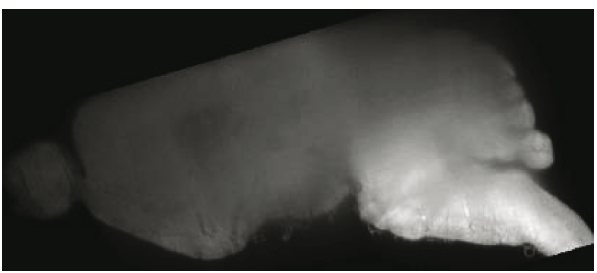

(b) Registered Template Volume

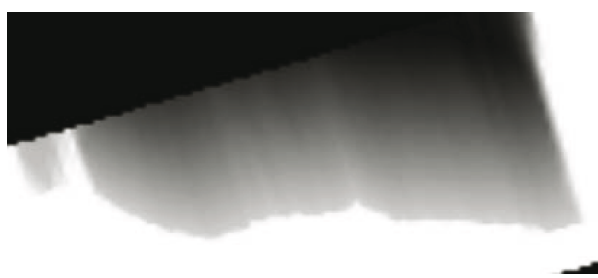

(d) Registered Template Quality

Fig. 3. Vertical slice of intensity and quality information from registered brain data

In order to incorporate the voxel quality information $Q_{R}$ and $Q_{T}$, we do not need to alter the registration algorithm itself. Only an adapted insertion of the voxel values with a weight $w_{i} \in[0 . .1]$ into equation 8 is needed:

$$
\begin{array}{r}
w_{i}=Q_{R}\left(\boldsymbol{x}_{\boldsymbol{i}}\right) Q_{T}\left(\phi\left(\boldsymbol{x}_{\boldsymbol{i}}\right)\right) \\
r_{i}^{*}=w_{i}\left(r_{i}-\bar{r}\right) ; \quad t_{i}^{*}=w_{i}\left(t_{i}-\bar{t}\right) \\
S^{*}=\frac{\sum_{i} r_{i}^{*} t_{i}^{*}}{\sqrt{\sum_{i} r_{i}^{* 2} \sum_{i} t_{i}^{* 2}}}
\end{array}
$$

Using this weighting, voxels with high quality in both volumes affect the individual sums of the NCC equation more. We denote this similarity measure Weighted Normalized Cross-Correlation (WNCC).

Note that a simple, approximative alternative is to use a limited joint volume of interest $\Omega$, where the quality is sufficiently high in both volumes. This is in our case a manually defined slab from the center slices. However, we would like to provide a general framework for incorporating quality information into registration rather than a quick specialized solution. In addition, the precision and especially robustness (as large portions of the images have to be omitted) of this center-slab approach is not convincing, as we experienced in an early registration study. Eventually, when the registered datasets are to be combined, the quality information is a prerequesite in order to allow a smooth transition. For merging two registered volumes, we consider the quality information in the following way:

$$
M\left(\boldsymbol{x}_{\boldsymbol{i}}\right)=\frac{R\left(\boldsymbol{x}_{\boldsymbol{i}}\right) Q_{R}\left(\boldsymbol{x}_{\boldsymbol{i}}\right)+T\left(\phi\left(\boldsymbol{x}_{\boldsymbol{i}}\right)\right) Q_{T}\left(\phi\left(\boldsymbol{x}_{\boldsymbol{i}}\right)\right)}{Q_{R}\left(\boldsymbol{x}_{\boldsymbol{i}}\right)+Q_{T}\left(\phi\left(\boldsymbol{x}_{\boldsymbol{i}}\right)\right)}
$$




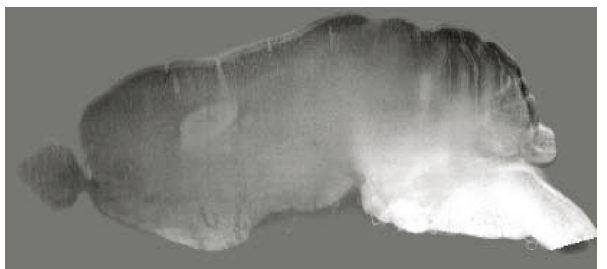

(a) Vertical Slice, NCC

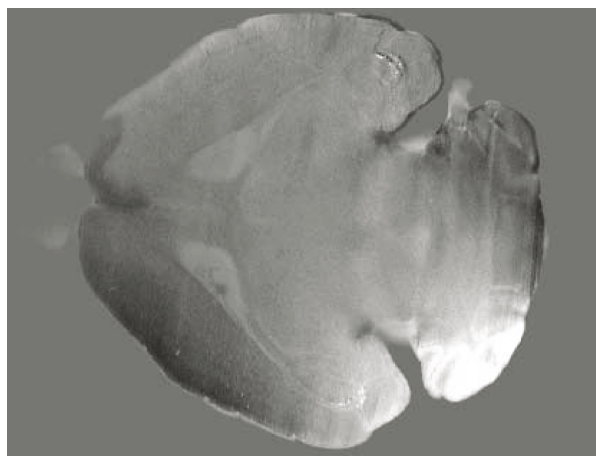

(c) Horizontal Slice, NCC

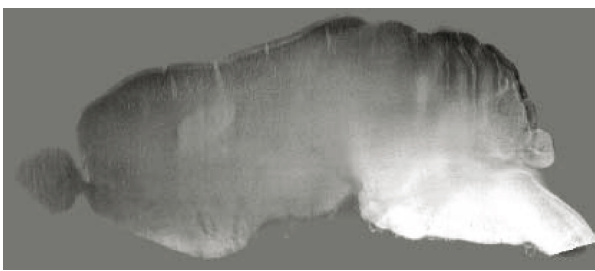

(b) Vertical Slice, WNCC

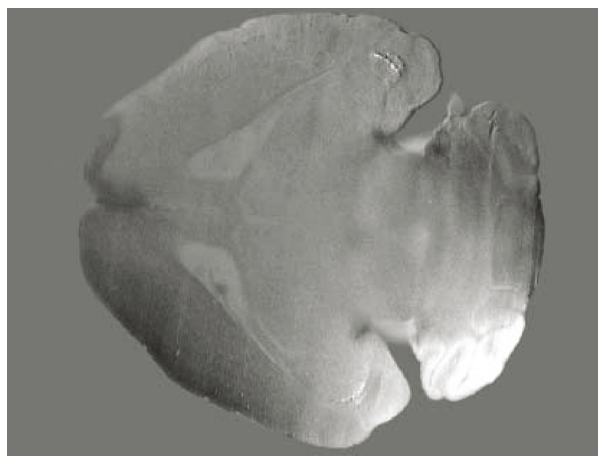

(d) Horizontal Slice, WNCC

Fig. 4. Difference of reference and template volumes of the brain preparation after registration. The background gray value indicates no error, dark and bright regions have larger intensity differences.

\section{Results}

\subsection{Registration Accuracy}

An in-vitro preparation of a mouse brain was imaged from the top and bottom side (figure 3). Its total length is $9 \mathrm{~mm}$, the volume was downsampled to size $256 x 256 x 189$ for registration. Figure 4 shows a vertical and horizontal difference slice for the two registration methods. The standard method results in larger errors on all borders, and especially a wrong displacement in vertical direction, as the blurred regions, located in opposite directions in both images, are fully considered for the similarity measure. The robustness of the registration was assessed with a randomized study: 236 registration computations were executed with initial transformations randomly displaced up to $1 \mathrm{~mm}$ and $6^{\circ}$ from the manually defined starting estimate. Both methods perform equally stable, the standard deviation of the resulting translational parameters is $6.4 \mu \mathrm{m}$, which corresponds to the parameter abortion criteria of the used Hill-Climbing optimizer. The mean translations of the two methods are $0.1 \mathrm{~mm}$ displaced (figure 5 ). This confirms the systematic bias due to blurring in opposite directions, which is compensated for by the weighted method. 




Fig. 5. Translation vectors of repeated registration from randomly displaced starting estimates. Blue $=\mathrm{NCC}$, Red $=$ WNCC.

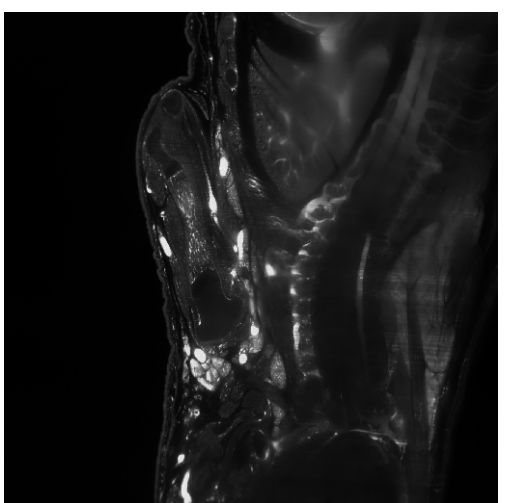

(a) single slice

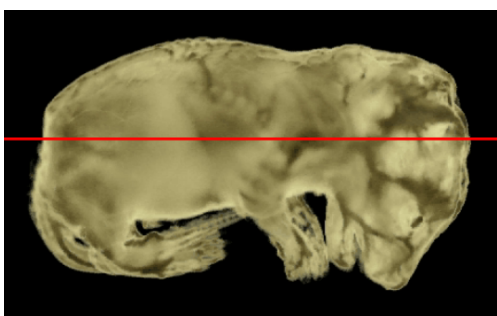

(c) VRT of single acquisition

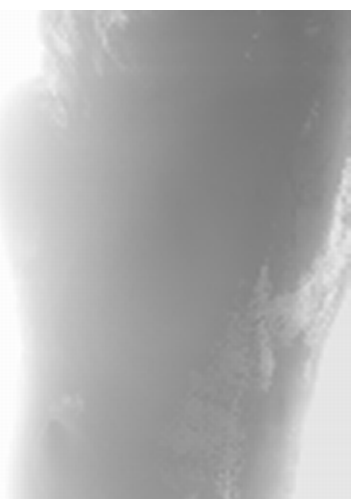

(b) quality slice

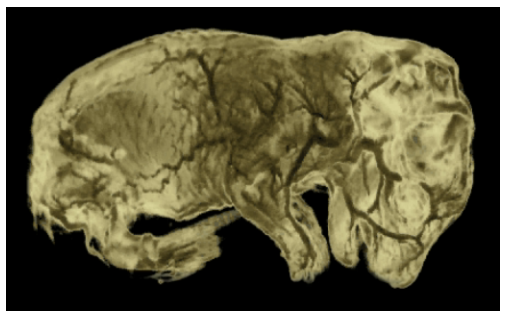

(d) VRT of reconstruction result

Fig. 6. (a) and (b) show the intensity and quality of a slice in the center of one of the embryo volumes. Volume rendering (VRT) of this volume is shown in (c), the red line indicates the approximate location of the slices (a) and (b). Volume rendering of the reconstruction result from two flipped acquisitions of a whole mouse embryo is depicted in $(\mathrm{d})$. 


\subsection{Merging}

Figure 6 shows the result of merging two volumes of a mouse embryo, the preparation was flipped sideways (approx. $180^{\circ}$ ) between the two acquisitions. Precise image registration is crucial, as the resulting voxels are taken from both volumes. Each single data set is heavily blurred on one side, while the final reconstruction in 6(d) depicts very sharp and detailed features throughout the whole volume without any visible reconstruction artifacts.

\section{Conclusion}

We presented an algorithm to reduce artifacts arising from a novel optical tomographic imaging modality. Depth-wise degradation of image quality can be overcome by registering multiple volumetric acquisitions. A physical simulation of the light scattering in the object allows us to derive additional volumes of relative voxel quality information. These are both used in an adapted registration algorithm, and for weighting multiple intensities during merging of the volumes. We believe that this straight-forward extension can be easily applied to other modalities where quality-related information is available. We demonstrated the increased precision of our quality-based registration on an optical tomography volume. The subsequent merging of registered data produces continuously high quality throughout the whole image space. The result are three-dimensional reconstructions of in-vitro biological tissue samples, with a resolution and quality which, to our knowledge, has never been achieved before.

\section{References}

1. Dodt, H.U., Leischner, U., Schierloh, A., Jährling, N., Mauch, C., Deininger, K., Deussing, J., Eder, M., Zieglgänsberger, W., Becker, K.: Ultramicroscoy: threedimensional visualization of neuronal networks in the whole mouse brain. Nature Methods 4, 331-336 (2007)

2. Spalteholz, W.: Über das Durchsichtigmachen von menschlichen und tierischen Präparaten und seine theoretischen Bedingungen. 2nd extended edn. S. Hirzel Leipzig Verlag (1914)

3. Williams, D.J.: The history of Werner Spalteholz's Handatlas der Anatomie des Menschen. Journal of Audiovisual Media in Medicine 22, 164-170 (1999)

4. Prahl, S.A., Keijzer, M., Jacques, S.L., Welch, A.J.: A Monte Carlo Model of Light Propagation in Tissue. SPIE Institute Series 5 (1989)

5. Maintz, J., Viergever, M.: A survey of medical image registration. Medical Image Analysis 2, 1-36 (1998) 